\title{
Original Articles Preoperative Hematological Parameters for Predicting Ovarian Torsion in Patients with Mature Cystic Teratoma
}

This article was published in the following Dove Press journal: International Journal of Women's Health

\author{
Jisun Lee' \\ Jinyoung Park ${ }^{2}$ \\ Hyun Jung Lee' \\ Mi Ju Kim' \\ Yoon Hee Lee ${ }^{3}$ \\ Gun Oh Chong ${ }^{3}$ \\ Dae Gy Hong ${ }^{3}$ \\ Taek Hoo Lee (D) \\ 'Department of Obstetrics and \\ Gynecology, School of Medicine, \\ Kyungpook National University, \\ Kyungpook National University Hospital, \\ Daegu, Republic of Korea; ${ }^{2}$ Department \\ of Surgery, School of Medicine, \\ Kyungpook National University, \\ Kyungpook National University Hospital, \\ Daegu, Republic of Korea; ${ }^{3}$ Department \\ of Obstetrics and Gynecology, School of \\ Medicine, Kyungpook National \\ University, Kyungpook National \\ University Chilgok Hospital, Daegu, \\ Republic of Korea
}

Correspondence: Taek Hoo Lee Department of Obstetrics \& Gynecology, I 30 Dongdeok-ro, Jung-gu, Daegu, 4I944, Republic of Korea

Tel +82-53-200-5735

Fax +82-53-423-7905

Email thlee@knu.ac.kr
Objective: The objective of this study is to evaluate the preoperative hematological parameters to predict ovarian torsion in patients with ovarian mature cystic teratoma. We also analyzed the diagnostic value of these makers to predict ovarian necrosis in cases of torsion as well as the effect of torsion on ovarian reserve.

Patients and Methods: This is a retrospective study of 132 patients who received either laparoscopic or laparotomy surgery for OMCT at a single university hospital. Clinical characteristics and preoperative hematological parameters were compared between patients with or without torsion. Patients with torsion were further classified as infarction and noninfarction group. Preoperative parameters were compared between the two groups as well. Results: White blood cell (WBC) count, neutrophil percent, neutrophil count, and neutrophil to lymphocyte ratio (NLR) were higher in the torsion group $(n=37)$ than the non-torsion group ( $\mathrm{n}=95) \quad(p<0.05$ for all). Although statistically insignificant, the preoperative antiMullerian hormone $(\mathrm{AMH})$ was lower in the torsion group than the non-torsion group $(4.07 \pm 3.38$ vs $6.1 \pm 3.6, p=0.122)$. In cases of torsion, the infarction group showed higher WBC count and lymphocyte count but lower hemoglobin level and platelet to lymphocyte ratio (PLR) than the non-infarction group ( $p<0.05$ for all).

Conclusion: The WBC count, neutrophil percent, neutrophil count, and NLR were higher in the cases of OMCT with torsion, and these parameters may be useful to diagnose OMCT with torsion. Also, adnexal torsion may deteriorate ovarian reserved as indicated by decreased AMH in torsion group.

Keywords: leukocyte counts, necrosis, ovarian neoplasm, ovarian teratoma, ovarian reserve, torsion

\section{Introduction}

Ovarian Mature cystic teratoma (OMCT) is a common benign tumor of the ovary accounting for approximately $60 \%$ of all primary benign ovarian tumors worldwide. OMCTs are composed of tissues derived from germ-cell layers such as endoderm (eg, lung, gastrointestinal tract), mesoderm (eg, muscle, urinary tract), and ectoderm (eg, skin, hair follicles, sebaceous glands). ${ }^{1}$ Most patients with OMCT are asymptomatic, but some experience abdominal distension, pelvic pain, or swelling depending on the size of the tumor. ${ }^{2,3}$

Among the complications associated with OMCT including rupture of cysts, infection, or malignant transformation, torsion is one of the most frequently observed conditions occurring in approximately $16 \%$ of patients. ${ }^{4}$ Torsion can 
occur in all ages with the highest incidence in the reproductive age. ${ }^{5}$ The most frequent classical symptom of ovarian torsion is the acute onset of lower abdominal pain accompanied by nausea and/or vomiting in women with adnexal tumor. ${ }^{6,7}$ However, they are not specific to adnexal torsion, but can also be found in other clinical conditions such as ovarian cyst rupture or pelvic inflammatory disease Consequently, it has been reported that only $38 \%$ of patients with adnexal torsion were preoperatively diagnosed. ${ }^{8}$

Early diagnosis of ovarian torsion is clinically very important because delay in the diagnosis may result in the loss of ovarian function consequently leading to the loss of fertility. Adnexal torsion occurs when the ovary rotates around its vascular axis and it is complicated by hemorrhagic infarction caused by the vascular or lymphatic blockage of ovarian parenchyma. ${ }^{9}$ The primary pathophysiology in ovarian torsion involves ischemia followed by reperfusion, which may result in the accumulation of the activated neutrophils that release reactive oxygen species (ROS). ${ }^{10}$ The ischemia due to torsion may initiate an inflammatory reaction resulting in increased levels of inflammatory parameters such as WBC count, neutrophil, and neutrophil to lymphocyte ratio (NLR). To aid the preoperative diagnosis of ovarian torsion, several inflammatory markers have been explored in association with ovarian torsion.

WBC count is an indicator of inflammation in the body. The five types of WBC include monocytes, lymphocytes, basophils, eosinophils, and neutrophils. Recently, the preoperative WBC count, neutrophil count, and NLR have been studied concerning adnexal torsion. ${ }^{11}$ Several studies are evaluating the preoperative diagnostic value of inflammatory markers for adnexal torsion, but most of the studies include adnexal torsion with various types of ovarian tumors. The inflammatory markers are greatly influenced by predisposing factors such as endometriosis, pregnancy, or autoimmune disease. Therefore, we sought to investigate various preoperative factors affecting torsion in patients with OMCT. The present study aimed to investigate whether the preoperative inflammatory markers might offer an accurate preoperative diagnosis of OMCT with torsion. We also investigated whether these serum parameters can predict ovarian necrosis in cases of ovarian torsion. The effect of ovarian torsion on the ovarian reserve is also included in the analysis.

\section{Patients and Methods}

Out of 187 patients who received adnexal surgery during the study period, the following number of patients were excluded from the analysis: 6 for adenomyosis, 5 for leiomyoma, 16 for tumor burst, 3 for inflammatory disease, 4 for malignancy, 14 for endometriosis, and 7 for pregnancy. A total of 132 patients with histological diagnosis of OMCT were included in this retrospective study. Clinical and hematological data were obtained from the patients who received either laparoscopic or laparotomy adnexal surgery at Kyungpook National University Hospital between January 2011 and March 2020 after obtaining approval from the center's institutional review board. The patients' consent was waived by the IRB board. The patient's data was treated with confidentiality and in compliance with the Declaration of Helsinki. The patients were classified either as an adnexal torsion group (37 patients) or a control group (95 patients) according to the surgical finding of adnexal torsion. The presence of necrosis, direction, and laterality of torsion were noted in the surgical evaluation.

All patients underwent either pelvic ultrasonography or abdominal computed tomography (CT) scan preoperatively. The size of the ovarian tumor was determined by the review of the operative records and imaging studies. Blood samples were also collected from the patients before the operation. Clinical and histopathological data were extracted from reviewing the medical records. Hematological parameters including WBC count, hemoglobin $(\mathrm{Hb})$, mean corpuscular volume (MCV), red cell distribution width (RDW), platelet count, mean platelet volume (MPV), neutrophil count, lymphocyte count, NLR, platelet to lymphocyte ratio (PLR), lymphocyte to monocyte ratio (LMR), anti-müllerian hormone (AMH), and tumor markers such as cancer antigen 125 (CA 125) and carbohydrate antigen 19-9 (CA 19-9) were included in the evaluation. The torsion group was further divided into the infarction and non-infarction groups according to the presence of infarction in the pathology reports. All the hematological and clinical characteristics were compared between the two groups.

The data were analyzed using SPSS version 25.0 (IBM, Armonk, NY, USA). The data was evaluated using the Kolmogorov-Smirnov test for normal distribution. Continuous variables were calculated as mean \pm standard deviation or mean (minimum-maximum) values and evaluated with either Student's $t$-test or Mann-Whitney test. Categorical variables were described as percentages and they were compared using $X^{2}$ or Fisher's exact test as appropriate. The ROC analysis was used to evaluate the diagnostic value of preoperative measurement of the inflammatory 
markers in predicting ovarian torsion. The area under the curve (AUC) was used to determine the sensitivity and specificity of each marker. The optimal cut-off values were calculated by ROC analysis. $P<0.05$ was considered statistically significant.

\section{Results}

A total of 132 patients were included in the study among which 37 patients were found to have OMCT with torsion and 95 patients without torsion. The clinical characteristics are presented in Table 1. The number of

Table I The Demographic Features and Hematological Parameters Between Torsion and Non-Torsion Groups

\begin{tabular}{|c|c|c|c|}
\hline Characteristics & Torsion (N=37) & Non-Torsion $(\mathbf{N}=95)$ & $p$-value \\
\hline Age (yrs.) & $24.19 \pm 14.9$ & $19.9 \pm 8.9$ & 0.058 \\
\hline Size $(\mathrm{cm})$ & $8.2 \pm 2.2$ & $8.1 \pm 3.9$ & 0.123 \\
\hline WBC count $\left(10^{3} / \mu \mathrm{L}\right)$ & $8.5(5.4-15.5)$ & $6.9(3.1-16.5)$ & $0.003^{\mathrm{a}}$ \\
\hline $\mathrm{Hb}(\mathrm{g} / \mathrm{dL})$ & $12.6 \pm 1.0$ & $12.9 \pm 1.2$ & 0.084 \\
\hline MCV (fl) & $85.0 \pm 4.8$ & $86.0 \pm 5.3$ & 0.463 \\
\hline RDW (\%) & $12.4(|1.4-| 4.3)$ & $12.6(\mid 1.3-17.1)$ & $0.529^{\mathrm{a}}$ \\
\hline PLT count $\left(10^{3} / \mu \mathrm{L}\right)$ & $295.7 \pm 64.2$ & $292.4 \pm 73.4$ & 0.916 \\
\hline MPV (fl) & $7.8 \pm 0.8$ & $7.8 \pm 1.1$ & 0.993 \\
\hline Neutrophil (\%) & $75.5(40.6-92.0)$ & $63.2(32.5-94.9)$ & $<0.001^{a}$ \\
\hline Neutrophil count $\left(10^{3} / \mu \mathrm{L}\right)$ & $6.70(2.2-13.2)$ & $4.37(1.2-13.7)$ & $0.002^{\mathrm{a}}$ \\
\hline Lymphocyte count $\left(10^{3} / \mu \mathrm{L}\right)$ & $1.42(0.6-3.5)$ & $1.75(0.5-32.4)$ & $0.003^{\mathrm{a}}$ \\
\hline Monocyte count $\left(10^{3} / \mu \mathrm{L}\right)$ & $0.25(0.08-0.67)$ & $0.33(0.09-3.8)$ & $0.026^{\mathrm{a}}$ \\
\hline NLR & $4.33(1.0-14.6)$ & $2.33(0.1-27.4)$ & $<0.00 I^{\mathrm{a}}$ \\
\hline PLR & $208(88.1-4 \mid 8.5)$ & 159 (4.8-82।.7) & $0.005^{\mathrm{a}}$ \\
\hline LMR & $6.5 \pm 3.9$ & $8.5 \pm 25.4$ & 0.635 \\
\hline CAI25 (U/mL) & $23.7 \pm 15.3$ & $25.4 \pm 21.8$ & 0.691 \\
\hline CAI9-9 (U/mL) & $141.9 \pm 196.0$ & $78.5 \pm 168.2$ & 0.229 \\
\hline AMH & $4.0 \pm 3.4$ & $6.2 \pm 3.6$ & 0.122 \\
\hline \multicolumn{4}{|l|}{ Laterality } \\
\hline Right (\%) & $16(43)$ & $52(55)$ & $0.235^{b}$ \\
\hline Left (\%) & $21(57)$ & $43(45)$ & $0.235^{\mathrm{b}}$ \\
\hline \multicolumn{4}{|l|}{ Operative method } \\
\hline Laparoscopy (\%) & $29(78)$ & $75(79)$ & $0.943^{b}$ \\
\hline Laparotomy (\%) & $8(22)$ & $20(2 I)$ & $0.943^{\mathrm{b}}$ \\
\hline \multicolumn{4}{|l|}{ Type of operation } \\
\hline Cystectomy (\%) & $21(57)$ & $78(82)$ & $0.004^{\mathrm{b}}$ \\
\hline Oophorectomy (\%) & $16(43)$ & $17(18)$ & $0.004^{b}$ \\
\hline
\end{tabular}

Notes: Values are mean \pm standard deviation, median (Min-Max) or absolute numbers (percentage). ${ }^{\mathrm{a}}$ Mann Whitney Test, ${ }^{\mathrm{b}} \mathrm{Chi}$-square test.

Abbreviations: WBC, white blood cell; Hb, Hemoglobin; MCV, mean corpuscular volume; RDW, red cell distribution width; MPV, mean platelet; NLR, neutrophil to lymphocyte ratio; PLR, platelet to lymphocyte ratio; LMR, lymphocyte to monocyte ratio; CA I25, cancer antigen I25; CA 19-9, carbohydrate antigen; AMH, anti-müllerian hormone. 
patients who received laparoscopic surgery was 29 (68\%) for the torsion group and 75 (79\%) for the nontorsion group. Moreover, the number of patients who received adnexal cystectomy was $21(57 \%)$ for the torsion group and $78(82 \%)$ for the non-torsion group. Salpingo-oophorectomy was performed in $16(43 \%)$ patients in the torsion group whereas 17 (18\%) patients received salpingo-oophorectomy in the non-torsion group. The type of surgery was performed according to the physician's preference. The pathological tumor size showed no significant difference between the two groups $(8.2 \pm 2.2 \mathrm{~cm}$ vs $8.1 \pm 3.9 \mathrm{~cm}, p=0.123)$. Although it was not statistically significant, patients in the torsion group were older than the patients in the non-torsion group $(24.19 \pm 14.9$ years vs $19.9 \pm 8.9$ years, $p=0.058)$. Preoperative hematological markers including $\mathrm{Hb}$, MCV, RDW, platelet count, MPV, LMR, and tumor markers (CA 125 and CA 19-9) showed no difference between the two groups.

Preoperative WBC count $\left(10^{3} / \mu \mathrm{L}\right)$, neutrophil percent (\%), neutrophil count $\left(10^{3} / \mu \mathrm{L}\right), \mathrm{NLR}$ and PLR were higher in patients with torsion compared to patients without torsion $\left(8.5 \times 10^{3} / \mu \mathrm{L}\right.$ vs $6.9 \times 10^{3} / \mu \mathrm{L}, p=0.003$; $75.5 \%$ vs. $63.2 \%, p<0.001 ; 6.7 \times 10^{3} / \mu \mathrm{L}$ vs $4.37 \times 10^{3} / \mu \mathrm{L}$, $p=0.002 ; 4.33$ vs $2.33, p<0.001 ; 208$ vs $159, p=0.005$ respectively). Lymphocyte count and monocyte count were lower in the torsion group than the non-torsion group $\left(1.42 \times 10^{3} / \mu \mathrm{L} \quad\right.$ vs $1.75 \times 10^{3} / \mu \mathrm{L}, \quad p=0.003$; $0.25 \times 10^{3} / \mu \mathrm{L}$ vs $0.33, p=0.026$ respectively). Although statistically insignificant, the preoperative AMH was lower in the torsion group than the non-torsion group $(4.07 \pm 3.38$ vs $6.1 \pm 3.6, p=0.122)$. When we evaluated these variables using multivariate analysis, only neutrophil percent was significantly associated with ovarian torsion as described in Table 3.

We further divided the patients with torsion into either infarction or non-infarction group according to the pathological evidence of necrosis or infarction. As depicted in Table 2, 6 patients showed infarction in the final pathologic reports whereas 31 patients showed no necrosis. Comparing the two groups, the patient's age, size of the tumor, MCV, RDW, platelet count, MPV, neutrophil count, monocyte count, NLR, LMR, AMH, and CA 125 were not different. Preoperative WBC count $\left(10^{3} / \mu \mathrm{L}\right)$ was higher in the infarction group than the non-infarction group $\left(11.54 \times 10^{3} / \mu \mathrm{L}\right.$ vs $\left.8.6 \times 10^{3} / \mu \mathrm{L}, p=0.028\right)$. The infarction group also showed a higher lymphocyte count than the non-infarction group $\left(2.1 \times 10^{3} / \mu \mathrm{L}\right.$ vs $\left.1.2 \times 10^{3} / \mu \mathrm{L}, p=0.036\right)$. Accordingly, the
PLR was lower in the infarction group than the noninfarction group (173.2 vs $286.2, p=0.05$ ). Interestingly, the preoperative $\mathrm{Hb}$ was lower in patients with infarction (12.1 g/ $\mathrm{dL}$ vs $13.1 \mathrm{~g} / \mathrm{dL}, p=0.012)$. When we evaluated these variables using multivariate analysis, WBC count was associated with infarction in adnexal torsion group (Table 4).

The AUC, cut-off values, sensitivity, and specificity of variables are described in Tables 5 and 6 . The cut-off values were $7360 \mu \mathrm{L}$ for $\mathrm{WBC}, 67.6 \%$ for neutrophil percent, $475010^{3} / \mu \mathrm{L}$ for neutrophil count, and 2.80 for NLR to predict OMCT with torsion. The ROC curve of affecting variables to diagnose OMCT with torsion is described in Figure 1. When these variables were examined for the infarction, the cut-off values for WBC count was $9730 \mu \mathrm{L}$ and $1910 \mu \mathrm{L}$ for lymphocyte count. The preoperative WBC count showed a higher cut-off value for predicting necrosis $(9730 \mu \mathrm{L})$ than for predicting OMCT with torsion $(7360 \mu \mathrm{L})$. It was also more sensitive to predict ovarian infarction in cases of torsion (sensitivity 83.3\%) than predicting OMCT torsion itself (sensitivity $82.9 \%$ ).

\section{Discussion}

Adnexal torsion is one of the few gynecological emergency cases with a prevalence of approximately $2 \%$ to $6 \%$. It was reported that about $3 \%$ of patients visiting the emergency department with acute abdominal pain have adnexal torsion. ${ }^{12,13}$ It can occur in patients of any age mostly common in women of reproductive age. OMCT is a common benign tumor of the ovary accounting for approximately $60 \%$ of all primary benign ovarian tumors worldwide. Approximately $16 \%$ of patients with OMCT have reported to have torsion. ${ }^{4}$

Ovarian torsion occurs when the ovary twists on the axis created between the infundibulopelvic ligament and the utero-ovarian ligament. The diagnosis is often made based on clinical symptoms along with laboratory or imaging findings. Clinical symptoms associated with ovarian torsion include acute onset of lower abdominal pain accompanied by nausea, vomiting, and/or low degree fever. ${ }^{14}$ However, these symptoms are not specific to ovarian torsion, and it is often difficult to differentiate among other problems such as ovarian cyst rupture, ectopic pregnancy, pelvic inflammatory disease, acute appendicitis, or gastroenteritis. ${ }^{15}$ It has been reported that only $38 \%$ of patients with adnexal torsion were preoperatively diagnosed with torsion. ${ }^{8}$ Early diagnosis and prompt treatment of ovarian torsion are very 
Table 2 The Demographic Features and Hematological Parameters Between Infarction and Non-Infarction Groups

\begin{tabular}{|c|c|c|c|}
\hline Characteristics & Infarction $(\mathbf{N}=6)$ & Non-Infarction $(\mathrm{N}=3 \mathrm{I})$ & $p$-value \\
\hline Age (yrs.) & $19.5(10-29)$ & $25(23-27)$ & $0.565^{\mathrm{a}}$ \\
\hline Size $(\mathrm{cm})$ & $7(7-7)$ & $9.75(9.5-10.0)$ & $0.917^{\mathrm{a}}$ \\
\hline WBC count $\left(10^{3} / \mu \mathrm{L}\right)$ & II.54 (10.4-12.6) & $8.6(8.0-9.2)$ & $0.028^{\mathrm{a}}$ \\
\hline $\mathrm{Hb}(\mathrm{g} / \mathrm{dL})$ & $12.1(12.1-12.2)$ & I3.I (13.0-13.2) & $0.012^{\mathrm{a}}$ \\
\hline $\mathrm{MCV}(\mathrm{fl})$ & $89.8(83.2-96.5)$ & $91.3(88.2-94.4)$ & $0.487^{\mathrm{a}}$ \\
\hline RDW (\%) & $12.9(12.4-13.4)$ & $12.7(12.6-12.9)$ & $0.254^{\mathrm{a}}$ \\
\hline PLT count $\left(10^{3} / \mu \mathrm{L}\right)$ & $352(336-368)$ & $330(326-335)$ & $0.976^{\mathrm{a}}$ \\
\hline MPV (fl) & $7.8(7.3-8.4)$ & $8.3(8.2-8.5)$ & $0.646^{\mathrm{a}}$ \\
\hline Neutrophil (\%) & 74.9 (7I.3-78.5) & $80.9(78.5-83.4)$ & $0.652^{\mathrm{a}}$ \\
\hline $\begin{array}{l}\text { Neutrophil count } \\
\left(10^{3} / \mu \mathrm{L}\right)\end{array}$ & $8.6(8.2-9.0)$ & $6.9(6.7-7.2)$ & $0.256^{\mathrm{a}}$ \\
\hline $\begin{array}{l}\text { Lymphocyte count } \\
\left(10^{3} / \mu \mathrm{L}\right)\end{array}$ & $2.1(1.5-2.8)$ & $1.2(0.9-1.5)$ & $0.036^{\mathrm{a}}$ \\
\hline $\begin{array}{l}\text { Monocyte count } \\
\left(10^{3} / \mu \mathrm{L}\right)\end{array}$ & $0.44(0.43-0.45)$ & $0.2(0.2-0.3$ & $0.350^{\mathrm{a}}$ \\
\hline NLR & $4.2(3.2-5.3)$ & $5.98(4.7-7.2)$ & $0.449^{\mathrm{a}}$ \\
\hline PLR & $173.2(129.6-216.8)$ & $286.2(221.9-350.6)$ & $0.050^{\mathrm{a}}$ \\
\hline LMR & $5.0(3.4-6.6)$ & $5.6(5.2-6.0)$ & $0.741^{\mathrm{a}}$ \\
\hline CAI25 (U/mL) & $29.4(7.9-50.8)$ & $23.6(22.4-24.7)$ & $0.206^{\mathrm{a}}$ \\
\hline AMH & $6.5(5.0-8.0)$ & $7.0(2.1-11.9)$ & $0.279^{\mathrm{a}}$ \\
\hline \multicolumn{4}{|l|}{ Laterality } \\
\hline Right (\%) & $2(33)$ & $14(45)$ & $0.68^{\mathrm{b}}$ \\
\hline Left (\%) & $4(67)$ & $17(55)$ & $0.68^{\mathrm{b}}$ \\
\hline \multicolumn{4}{|l|}{ Operative method } \\
\hline Laparoscopy (\%) & $4(66)$ & $25(80)$ & $0.47^{b}$ \\
\hline Laparotomy (\%) & $2(34)$ & $6(20)$ & $0.47^{b}$ \\
\hline $\begin{array}{l}\text { Type of operation } \\
\text { Cystectomy (\%) }\end{array}$ & 0 & $10(32)$ & $0.002^{b}$ \\
\hline Oophorectomy (\%) & $6(100)$ & $21(68)$ & $0.002^{\mathrm{b}}$ \\
\hline
\end{tabular}

Notes: Values are mean \pm standard deviation, median (Min-Max), or absolute numbers (percentage). ${ }^{\mathrm{a}}$ Mann Whitney Test, ${ }^{\mathrm{b}}$ Fisher's exact test.

Abbreviations: WBC, white blood cell; Hb, Hemoglobin; MCV, mean corpuscular volume; RDW, red cell distribution width; MPV, mean platelet; NLR, neutrophil to lymphocyte ratio; PLR, platelet to lymphocyte ratio; LMR, lymphocyte to monocyte ratio; CA I25, cancer antigen I25; CA I9-9, carbohydrate antigen; AMH, anti-müllerian hormone.

important to prevent loss of ovarian function and to preserve fertility.

There have been various studies evaluating the preoperative imaging modalities and laboratory parameters to support the diagnosis of ovarian torsion.
Ultrasonography is most commonly used for the diagnosis of ovarian torsion in an emergency setting. The common findings of adnexal torsion include a cystic, solid, or complex mass with thickened walls and internal hemorrhage. ${ }^{16}$ Decreased or absent doppler flow in 
Table 3 Logistic Regression Model of WBC Count, Neutrophil Percent, Neutrophil Count, and Neutrophil to Lymphocyte Ratio with Torsion on Mature Cystic Teratomas

\begin{tabular}{|l|l|l|l|l|}
\hline \multirow{2}{*}{ Characteristics } & \multicolumn{2}{l|}{ Univariate Analysis } & \multicolumn{2}{l|}{ Multivariate Analysis } \\
\cline { 2 - 5 } & OR (95\% CI) & p-value & OR (95\% Cl) & p-value \\
\hline WBC count $\left(10^{3} / \mu \mathrm{L}\right)$ & $1.142(1.007-1.295)$ & 0.039 & & 0.001 \\
\hline Neutrophil $(\%)$ & $1.078(1.039-1.118)$ & $<0.001$ & $0.741(0.618-0.888)$ & \\
\hline Neutrophil count $\left(10^{3} / \mu \mathrm{L}\right)$ & $1.000(1.000-1.000)$ & 0.004 & & \\
\hline NLR & $1.096(1.001-1.199)$ & 0.046 & & \\
\hline
\end{tabular}

Abbreviations: WBC, white blood cell; NLR, neutrophil to lymphocyte ratio; OR, odds ratio; Cl, confidence interval.

Table 4 Logistic Regression Model of WBC Count, Hemoglobin, Lymphocyte Count, Platelet to Lymphocyte Ratio for Infarction in Torsion

\begin{tabular}{|l|l|l|l|l|}
\hline \multirow{2}{*}{ Characteristics } & Univariate Analysis & & \multicolumn{2}{l|}{ Multivariate Analysis } \\
\cline { 2 - 5 } & OR (95\% Cl) & p-value & OR (95\% CI) & p-value \\
\hline WBC count $\left(10^{3} / \mu \mathrm{L}\right)$ & $1.459(1.008-2.11)$ & 0.045 & $1.97(1.03-3.74)$ & 0.039 \\
\hline $\mathrm{Hb}(\mathrm{g} / \mathrm{dL})$ & $0.330(0.125-0.870)$ & 0.025 & $0.26(0.078-0.894)$ & 0.032 \\
\hline Lymphocyte count $\left(10^{3} / \mu \mathrm{L}\right)$ & $6.014(1.263-28.64)$ & 0.024 & & \\
\hline PLR & $0.984(0.967-1.001)$ & 0.068 & & \\
\hline
\end{tabular}

Abbreviations: WBC, white blood cell; Hb, hemoglobin; PLR, platelet to lymphocyte ratio; OR, odds ratio; $\mathrm{Cl}$, confidence interval.

the ovarian vessel may be observed in the case of the adnexal torsion. The ultrasonographic findings and the absence of Doppler flow however do not show high diagnostic value. Approximately $45 \%$ of patients with ovarian torsion had normal preoperative ultrasonographic findings and Doppler ultrasonography has been reported to be normal in up to $60 \%$ of ovarian torsion cases. ${ }^{7,17}$ Accordingly, preoperative ultrasonography alone is not reliable for the diagnosis of ovarian torsion.

Another imaging modality commonly used in evaluating ovarian torsion is an abdominal CT scan. In cases of adnexal torsion, CT findings show uterine deviation toward the side of the affected ovary and thickened pedicle. ${ }^{18}$ The specificity of CT in the diagnosis of adnexal torsion ranges from $74 \%$ to $91 \%$ with a low positive predictive value of $46.1 \% .{ }^{19,20}$ Despite the usefulness of CT scan in the evaluation of adnexal torsion, it is often not available in underdeveloped countries, and the interpretations of CT findings may depend on the radiologists.

Recently, preoperative laboratory parameters especially hematological inflammatory markers have been studied in the evaluation of adnexal torsion. The primary pathophysiology in ovarian torsion involves ischemia followed by reperfusion, which may result in the accumulation of the activated neutrophils that release reactive oxygen species

Table 5 Diagnostic Values of Affecting Variables for Mature Cystic Teratoma with Torsion

\begin{tabular}{|l|l|l|l|l|l|}
\hline Variables & AUC $(95 \% \mathbf{~ C l})$ & p-value & Cut-Off Value & Sensitivity (\%) & Specificity (\%) \\
\hline WBC count $\left(10^{3} / \mu \mathrm{L}\right)$ & $0.693(0.602-0.784)$ & 0.001 & 7.36 & 82.9 & 62.4 \\
\hline Neutrophil $(\%)$ & $0.764(0.675-0.852)$ & $<0.001$ & 67.6 & 88.6 & 62.4 \\
\hline Neutrophil count $\left(10^{3} / \mu \mathrm{L}\right)$ & $0.723(0.634-0.812)$ & $<0.001$ & 4.75 & 88.6 & 63.4 \\
\hline NLR & $0.751(0.661-0.840)$ & $<0.001$ & 2.80 & 88.6 & 64.5 \\
\hline
\end{tabular}

Abbreviations: WBC, white blood cell; NLR, neutrophil to lymphocyte ratio, AUC, area under the curve; Cl, confidence interval. 
Table 6 Diagnostic Values of Affecting Variables for Necrosis in OMCT with Torsion

\begin{tabular}{|l|l|l|l|l|l|}
\hline Variables & AUC (95\% Cl) & P-value & Cut-Off Value & Sensitivity (\%) & Specificity (\%) \\
\hline WBC count $\left(10^{3} / \mu \mathrm{L}\right)$ & $0.805(0.653-0.956)$ & 0.020 & 9.73 & 83.3 & 75.9 \\
\hline $\mathrm{Hb}(\mathrm{g} / \mathrm{dL})$ & $0.233(0.011-0.454)$ & 0.042 & 10.25 & 100 & 3.4 \\
\hline Lymphocyte count $\left(10^{3} / \mu \mathrm{L}\right)$ & $0.773(0.512-1.00)$ & 0.038 & 1.91 & 66.7 & 89.7 \\
\hline PLR & $0.253(0.027-0.479)$ & 0.060 & 87.1 & 100 & 0 \\
\hline
\end{tabular}

Abbreviations: WBC, white blood cell; $\mathrm{Hb}$, hemoglobin; PLR, platelet to lymphocyte ratio; $\mathrm{AUC}$, area under the curve; $\mathrm{Cl}$, confidence interval.

(ROS). ${ }^{10}$ Yigiter et al evaluated the biochemical and histopathological changes in rat ovaries concerning experimental ischemia and ischemia/reperfusion injury. They found that ovarian tissues in the ischemia group showed histopathological changes such as hemorrhage, cellular degeneration, along with the acute inflammatory processes such as neutrophil adhesion and migration, degenerative cells, stromal edema, and hemorrhage. ${ }^{10}$

In this study, we compared the preoperative inflammatory markers between the ovarian torsion and non-torsion group. The torsion group showed significantly higher preoperative WBC count, neutrophil count, and NLR while having lower lymphocyte and monocyte count compared to the nontorsion group. Ercan et al also found that both WBC and neutrophil counts were increased in adnexal torsion patients whereas the lymphocyte level was lower than those of the control group. ${ }^{21}$

NLR is another important inflammatory indicator, which has been widely evaluated in association with cardiovascular diseases, diabetes mellitus, autoimmune diseases, and several gynecological diseases including ovarian cancer, cervical cancer, endometriosis, and pelvic inflammatory disease..$^{22-28}$ In a study by Lee et al, 14 out of 24 patients with OMCT with torsion showed higher NLR value compared to patients without torsion. ${ }^{29}$ The results of recent studies collectively suggest that NLR may be a valuable marker for diagnosing adnexal torsion. When comparing the diagnostic value of WBC count and NLR, many studies indicate that NLR is superior to WBC count to evaluate the inflammatory processes in surgical
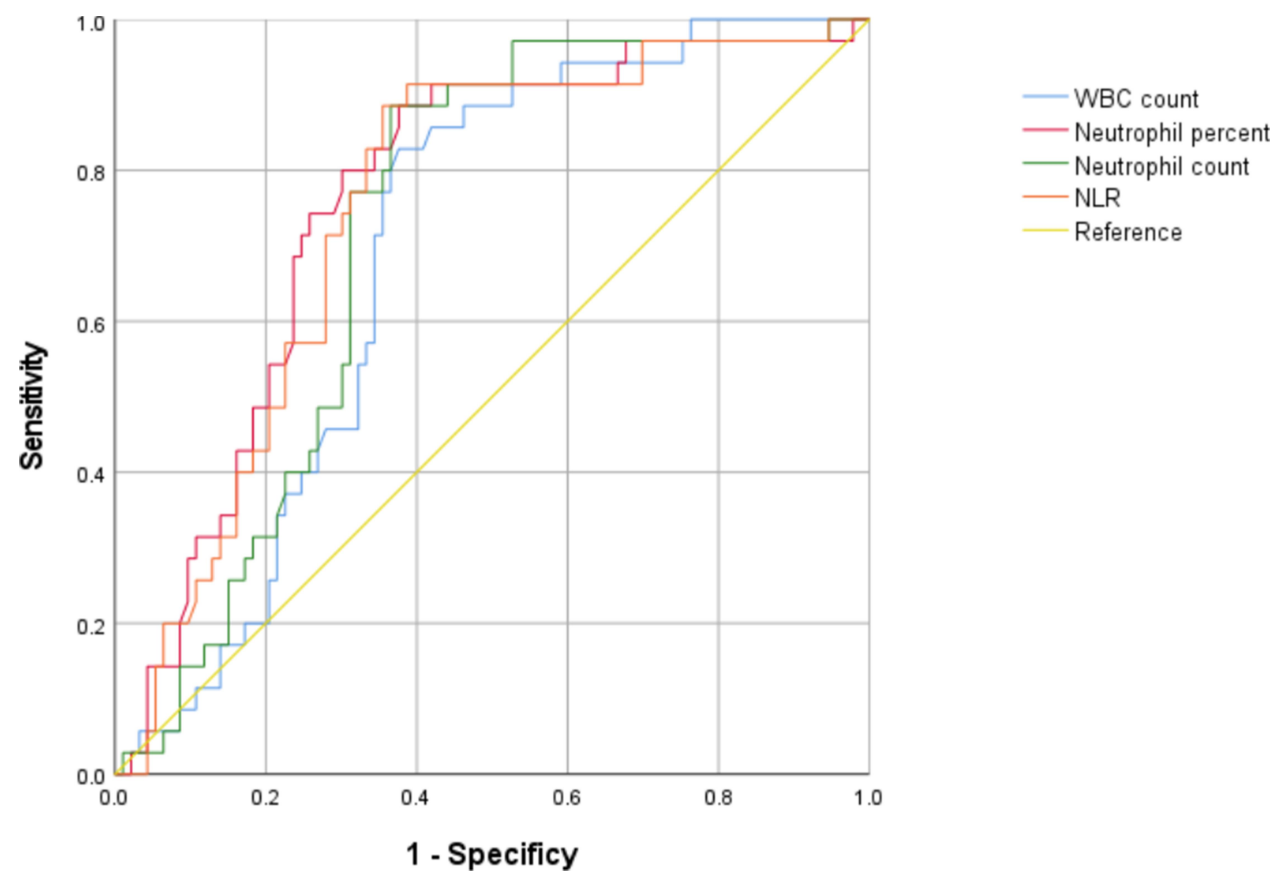

Reference

Figure I Receiver operating curve of affecting variables to diagnose mature ovarian teratoma with torsion. Combination consists of WBC count, neutrophil percent, neutrophil count, and NLR.

Abbreviations: WBC, white blood cells; NLR, neutrophil to lymphocyte ratio. 
conditions. ${ }^{30}$ In a study by Ercan et al, the sensitivity and specificity of NLR were $88.9 \%$ and $100 \%$ respectively whereas WBC count showed $74.1 \%$ sensitivity and $83.3 \%$ specificity for diagnosing ovarian torsion. ${ }^{21}$ Our study also showed similar results that NLR had higher sensitivity of $88.6 \%$ and specificity of $64.5 \%$ than those of WBC count (sensitivity $82.9 \%$, specificity $62.4 \%$ ) in predicting ovarian torsion. Furthermore, NLR was higher in AUC value (0.75) compared to WBC count (0.69), which indicates that NLR is more sensitive than WBC count in diagnosing adnexal torsion. The cut-off values for predicting OMCT with torsion were 2.80 for NLR, $7360 / \mu \mathrm{L}$ for WBC count, $67.6 \%$ for neutrophil percent, and $4750 / \mu \mathrm{L}$ for neutrophil count, which were similar to previously published studies. $^{21,29}$ Also, preoperative neutrophil count showed diagnostic value similar to NLR (sensitivity $88.6 \%$, specificity $63.8 \%$ ). According to our study, preoperative neutrophil percent, neutrophil count, and NLR are more sensitive than WBC count in predicting OMCT with torsion.

Despite the abundant studies on the possible diagnostic values of preoperative inflammatory markers for ovarian torsion, it has not been reported whether preoperative inflammatory markers are associated with ovarian necrosis in ovarian torsion. NLR was previously evaluated in patients with myocardial ischemia, in which the NLR was found to be increased in acute cases. ${ }^{31} \mathrm{We}$ hypothesized that WBC count, NLR, and other inflammatory markers would be significantly higher in patients with necrosis due to the ischemic nature of adnexal torsion. In this study, patients with necrosis had higher preoperative WBC count and lymphocyte count, but lower PLR value when compared to those without necrosis. The preoperative WBC count was associated both with adnexal torsion in patients with OMCT, and ovarian infarction among patients with torsion. The cut-off value of WBC count was $9730 / \mu \mathrm{L}$ with $83.3 \%$ sensitivity and $75.9 \%$ specificity for predicting torsion whereas the cut-off value was $7360 / \mu \mathrm{L}$ with a sensitivity of $82.9 \%$ and specificity of $62.4 \%$ for predicting necrosis in the torsion group. This increased cut-off value for predicting necrosis may indicated that ovarian necrosis may cause more inflammatory changes than adnexal torsion itself.

The absolute lymphocyte count is a simple immunological index that reflects the general change of the immune system. The association between the lymphocyte count and necrosis was evaluated in patients with acute pancreatitis. In a study by Shen et al, the lymphocyte count was significantly associated with the development of infected pancreatic necrosis. $^{32}$ The pre-operative lymphocyte count was also evaluated in patients with adnexal torsion and several studies showed no significant diagnostic value. ${ }^{29}$ According to our study, pre-operative lymphocyte count was lower in the torsion group compared to the nontorsion group, but it was higher in the infarction group than the non-infarction group.

A possible explanation for such results may be attributed to the oxidative stress found in ovarian tissue with torsion. As previously mentioned, the mechanism of tissue damage is attributed to the direct damage caused by ischemia during torsion as found in other organs such as the kidney or brain. ${ }^{33}$ The damaged tissues produce excessive amounts of ROS and reactive nitrogen species (RNS), which lead to leukocyte activation resulting in further damage and cell death. ${ }^{34}$ In a study by Uguralp et al, levels of malondialdehyde (MDA) and nitric oxide (NO) were higher in the ischemic ovarian tissue, which may, in turn, induce lymphocyte activation. ${ }^{35}$ This mechanism may reflect increased lymphocyte count in the infarction group of patients.

Interestingly, the patient with infarction showed lower serum $\mathrm{Hb}$ levels than patients without infarction. There are conflicting results regarding the association between $\mathrm{Hb}$ and clinical outcomes. Concerning ovarian torsion, there is scarce evidence for the diagnostic value of preoperative $\mathrm{Hb}$ for predicting ovarian torsion including ovarian infarction. Although patients with the infarction group showed a lower $\mathrm{Hb}$ level than the non-infarction group in our study, the AUC was only 0.233 and had very low specificity $(3.4 \%)$. The result of our study implies the preoperative $\mathrm{Hb}$ level may have some association with ovarian necrosis in cases of ovarian torsion. However, further study is required to make a more concrete conclusion on the association between $\mathrm{Hb}$ and ovarian infarction.

Tumor markers such as CA 125 and CA 19-9 have also been considered as possible predictive markers for ovarian torsion. Wang et al examined the efficacy of CA 125, CA 19-9, and NLR as markers for the diagnosis of MCT with torsion. They found that torsion was associated with abnormally high levels of CA 19-9, but it was correlated with the size of the ovarian tumor being at least $8.5 \mathrm{~cm}$ in diameter. ${ }^{36}$ Our study showed no difference in terms of the ovarian tumor, and accordingly the preoperative CA 125 and CA 19-9 levels were similar between the torsion and non-torsion groups. As Var et al concluded in their study, the most important parameter affecting CA 
19-9 elevation in the OMCT is the tumor size. ${ }^{37}$ The preoperative CA 125 and CA 19-9 levels are probably related to the size of the ovarian tumor rather than the adnexal torsion itself.

Our study has clinical importance in that it included only patients with OMCT. Different histopathology of ovarian tumors may influence serum inflammatory parameters such as endometriosis causing increased WBC count, neutrophil count, NLR, and MPV. ${ }^{27}$ We excluded pregnancy patients and patients with endometriosis to eliminate possible variables affecting the preoperative levels of serum parameters. These findings of our study may be applied to patients with OMCT who are in suspicion of adnexal torsion. It also supports the previous findings that the preoperative WBC count, neutrophil count, and NLR are associated with ovarian torsion. The sensitivity of WBC count, NLR, and neutrophil count $(82.9 \%$, $88.6 \%$, and $88.6 \%$, respectively) were found to be higher than that of $\mathrm{CT}(75 \%)$ in predicting ovarian torsion. Consequently, when a patient visits a hospital with acute lower abdominal pain and is suspicious of adnexal torsion, these simple and easily obtained preoperative serum markers can be useful especially in emergency settings where an expensive CT scan is not available. Another important finding of our study is ovarian necrosis is associated with increased WBC count among ovarian torsion patients. The cut-off value for necrosis $(9730 / \mu \mathrm{L})$ was higher than for the torsion $(7360 / \mu \mathrm{L})$. The increased WBC count may alert physicians to institute prompt treatment for ovarian torsion to preserve ovarian function.

The present study has several limitations. The sample size is too small especially for the evaluation of the ovarian necrosis population. The retrospective design of the study also suggests only a possible correlation between the preoperative parameters and torsion. Further prospective studies are required to confirm the diagnostic value of preoperative markers in OMCT with torsion.

The clinical presentations of patients were not included in the study as all patients in the torsion group experienced unspecified abdominal pain. The clinical symptoms were not well described in the medical chart. It would be interesting to find the preoperative parameters to predict ovarian torsion in non-symptomatic patients. Moreover, one of the most important factors associated with ovarian necrosis is the time interval between the onset of the symptom and the initiation of treatment as well as the time interval between the measurement of preoperative hematological parameters and the start of the operation. Unfortunately, the time interval between these variables were not available. Since our institution is a University-based hospital, the number of patients coming to the emergency room is overwhelming. Moreover, we do not have enough sources to carry on the operation as quickly as possible for many practical reasons such as shortage of anesthesiology doctors and shortage of physicians available for operation. The time interval was not recorded in this study, which may have influenced the results. Further investigation including the ovarian reserve marker as well as the time interval may be required to strengthen the results of our study.

\section{Conclusions}

In conclusion, the present study demonstrated that preoperative WBC count, neutrophil count, and NLR can be useful preoperative markers in predicting OMCT with torsion especially WBC count being involved in the necrosis associated with torsion. Along with clinical symptoms, applications of these preoperative measurements could support the preoperative identification of adnexal torsion.

\section{Disclosure}

The authors report no conflicts of interest in this work.

\section{References}

1. Ozgur T, Atik E, Silfeler DB, Toprak S. Mature cystic teratomas in our series with review of the literature and retrospective analysis. Arch Gynecol Obstet. 2012;285(4):1099-1101. doi:10.1007/s00404011-2171-8

2. Comerci JT, Licciardi F, Bergh PA, Gregori C, Breen JL. Mature cystic teratoma: a clinicopathologic evaluation of 517 cases and review of the literature. Obstet Gynecol. 1994;84(1):22-28.

3. Peterson WF, Prevost EC, Edmunds FT, Hundley JM, Morris FK. Benign cystic teratomas of the ovary; a clinico-statistical study of 1007 cases with a review of the literature. Am J Obstet Gynecol. 1955;70(2):368-382. doi:10.1016/S0002-9378(16)37681-5

4. Artunc Ulkumen B, Goker A, Pala HG, Ordu S. Abnormal elevated CA 19-9 in the dermoid cyst: a sign of the ovarian torsion? Case Rep Obstet Gynecol. 2013;2013:860505. doi:10.1155/2013/860505

5. White M, Stella J. Ovarian torsion: 10-year perspective. Emerg Med Australas. 2005;17(3):231-237. doi:10.1111/j.17426723.2005.00728.x

6. Kokoska ER, Keller MS, Weber TR. Acute ovarian torsion in children. Am J Surg. 2000;180(6):462-465. doi:10.1016/S0002-9610(00)00503-1

7. Oltmann SC, Fischer A, Barber R, Huang R, Hicks B, Garcia N. Cannot exclude torsion-a 15-year review. J Pediatr Surg. 2009;44 (6):1212-1216; discussion 1217. doi:10.1016/j.jpedsurg.2009.02.028

8. Argenta PA, Yeagley TJ, Ott G, Sondheimer SJ. Torsion of the uterine adnexa. pathologic correlations and current management trends. J Reprod Med. 2000;45(10):831-836.

9. Graif M, Itzchak Y. Sonographic evaluation of ovarian torsion in childhood and adolescence. AJR Am J Roentgenol. 1988;150 (3):647-649. doi:10.2214/ajr.150.3.647

10. Yigiter M, Halici Z, Odabasoglu F, et al. Growth hormone reduces tissue damage in rat ovaries subjected to torsion and detorsion: biochemical and histopathologic evaluation. Eur J Obstet Gynecol Reprod Biol. 2011;157(1):94-100. doi:10.1016/j.ejogrb.2011.02.012 
11. Tobiume T, Shiota M, Umemoto M, Kotani Y, Hoshiai H. Predictive factors for ovarian necrosis in torsion of ovarian tumor. Tohoku J Exp Med. 2011;225(3):211-214. doi:10.1620/tjem.225.211

12. Sasaki KJ, Miller CE. Adnexal torsion: review of the literature. J Minim Invasive Gynecol. 2014;21(2):196-202. doi:10.1016/j.jmig.2013.09.010

13. Oelsner G, Shashar D. Adnexal torsion. Clin Obstet Gynecol. 2006;49(3):459-463. doi:10.1097/00003081-200609000-00006

14. Shadinger LL, Andreotti RF, Kurian RL. Preoperative sonographic and clinical characteristics as predictors of ovarian torsion. J Ultrasound Med. 2008;27(1):7-13. doi:10.7863/jum.2008.27.1.7

15. Nur Azurah AG, Zainol ZW, Zainuddin AA, Lim PS, Sulaiman AS, $\mathrm{Ng}$ BK. Update on the management of ovarian torsion in children and adolescents. World J Pediatr. 2015;11(1):35-40. doi:10.1007/s12519014-0536-3

16. Rha SE, Byun JY, Jung SE, et al. CT and MR imaging features of adnexal torsion. Radiographics. 2002;22(2):283-294. doi:10.1148/ radiographics.22.2.g02mr02283

17. Servaes S, Zurakowski D, Laufer MR, Feins N, Chow JS. Sonographic findings of ovarian torsion in children. Pediatr Radiol. 2007;37(5):446-451. doi:10.1007/s00247-007-0429-x

18. Lee MS, Moon MH, Woo H. CT findings of adnexal torsion: a matched case-control study. PLoS One. 2018;13(7):e0200190. doi:10.1371/journal.pone.0200190

19. Jung SI, Park HS, Yim Y, et al. Added value of using a CT coronal reformation to diagnose adnexal torsion. Korean J Radiol. 2015;16 (4):835-845. doi:10.3348/kjr.2015.16.4.835

20. Bar-On S, Mashiach R, Stockheim D, et al. Emergency laparoscopy for suspected ovarian torsion: are we too hasty to operate? Fertil Steril. 2010;93(6):2012-2015. doi:10.1016/j.fertnstert.2008.12.022

21. Ercan O, Kostu B, Bakacak M, Coskun B, Tohma A, Mavigok E. Neutrophil to Lymphocyte ratio in the diagnosis of adnexal torsion. Int J Clin Exp Med. 2015;8(9):16095-16100.

22. Angkananard T, Anothaisintawee T, McEvoy M, Attia J, Thakkinstian A. Neutrophil lymphocyte ratio and cardiovascular disease risk: a systematic review and meta-analysis. Biomed Res Int. 2018;2018:2703518. doi:10.1155/2018/2703518

23. Xu T, Weng Z, Pei C, et al. The relationship between neutrophil-tolymphocyte ratio and diabetic peripheral neuropathy in Type 2 diabetes mellitus. Medicine. 2017;96(45):e8289. doi:10.1097/ MD.0000000000008289

24. Djaballah-Ider F, Touil-Boukoffa C. Effect of combined colchicinecorticosteroid treatment on neutrophil/lymphocyte ratio: a predictive marker in Behçet disease activity. Inflammopharmacology. 2020;28 (4):819-829. doi:10.1007/s10787-020-00701-X

25. Chen G, Zhu L, Yang Y, Long Y, Li X, Wang Y. Prognostic role of neutrophil to lymphocyte ratio in ovarian cancer: a meta-analysis. Technol Cancer Res Treat. 2018;17:1533033818791500. doi:10.1177/ 1533033818791500
26. Prabawa IPY, Bhargah A, Liwang F, et al. Pretreatment Neutrophil-to-Lymphocyte ratio (NLR) and Platelet-to-Lymphocyte Ratio (PLR) as a predictive value of hematological markers in cervical cancer. Asian Pac J Cancer Prev. 2019;20(3):863-868. doi:10.31557/APJCP.2019.20.3.863

27. Turgut A, Hocaoglu M, Ozdamar O, Usta A, Gunay T, Akdeniz E. Could hematologic parameters be useful biomarkers for the diagnosis of endometriosis? Bratisl Lek Listy. 2019;120(12):912-918. doi:10.4149/BLL_2019_153

28. Alay I, Kaya C, Karaca I, et al. The effectiveness of neutrophil to lymphocyte ratio in prediction of medical treatment failure for tubo-ovarian abscess. J Obstet Gynaecol Res. 2019;45 (6):1183-1189. doi:10.1111/jog.13946

29. Lee JY, Shin W, Kim JS, Park JH, Cho S. Combination of clinical and laboratory characteristics may serve as a potential diagnostic marker for torsion on mature cystic teratomas. Obstet Gynecol Sci. 2018;61 (3):386-394. doi:10.5468/ogs.2018.61.3.386

30. Zahorec R. Ratio of neutrophil to lymphocyte counts-rapid and simple parameter of systemic inflammation and stress in critically ill. Bratisl Lek Listy. 2001;102(1):5-14.

31. Bhat T, Teli S, Rijal J, et al. Neutrophil to lymphocyte ratio and cardiovascular diseases: a review. Expert Rev Cardiovasc Ther. 2013;11(1):55-59. doi:10.1586/erc.12.159

32. Shen X, Sun J, Ke L, et al. Reduced lymphocyte count as an early marker for predicting infected pancreatic necrosis. $B M C$ Gastroenterol. 2015;15(1):147. doi:10.1186/s12876-015-0375-2

33. White BC, Sullivan JM, DeGracia DJ, et al. Brain ischemia and reperfusion: molecular mechanisms of neuronal injury. J Neurol Sci. 2000;179(S1-2):1-33.

34. Grace PA. Ischaemia-reperfusion injury. $B r \quad J$ Surg. 1994;81 (5):637-647. doi:10.1002/bjs.1800810504

35. Uguralp S, Bay Karabulut A, Mizrak B. Effects of pentoxifylline and vitamin $\mathrm{E}$ on the bilateral ovary after experimental ovarian ischemia. Eur J Pediatr Surg. 2005;15(2):107-113. doi:10.1055/s-2004-821256

36. Wang YQ, Xia WT, Wang F, Zhuang XX, Zheng FY, Lin F. Use of cancer antigen 125, cancer antigen 19-9, and the neutrophil-tolymphocyte ratio to diagnose mature cystic teratoma with torsion. Int J Gynaecol Obstet. 2017;137(3):332-337. doi:10.1002/ijgo.12139

37. Var T, Tonguc EA, Ugur M, Altinbas S, Tokmak A. Tumor markers panel and tumor size of ovarian dermoid tumors in reproductive age. Bratisl Lek Listy. 2012;113(2):95-98. doi:10.4149/bll_2012_022

\section{Publish your work in this journal}

The International Journal of Women's Health is an international, peerreviewed open-access journal publishing original research, reports, editorials, reviews and commentaries on all aspects of women's healthcare including gynecology, obstetrics, and breast cancer. The manuscript management system is completely online and includes a very quick and fair peer-review system, which is all easy to use. Visit http://www.dovepress.com/testimonials.php to read real quotes from published authors. 